\title{
Médiévales
}

Langues, Textes, Histoire

45 | automne 2003

Grammaires du vulgaire

\section{Le rôle des traductions médiévales dans l'évolution de la langue française et la constitution de sa grammaire}

The Role of Mediaeval Translations in the Evolution of the French Language and the Establishment of its Grammar

\section{Claude Buridant}

\section{OpenEdition}

\section{Journals}

Édition électronique

URL : https://journals.openedition.org/medievales/637

DOI : 10.4000/medievales. 637

ISSN : $1777-5892$

\section{Éditeur}

Presses universitaires de Vincennes

\section{Édition imprimée}

Date de publication : 16 septembre 2003

Pagination : 67-84

ISBN : 2-84292-146-1

ISSN : 0751-2708

\section{Référence électronique}

Claude Buridant, « Le rôle des traductions médiévales dans l'évolution de la langue française et la constitution de sa grammaire », Médiévales [En ligne], 45 | automne 2003, mis en ligne le 13 mars 2006, consulté le 23 avril 2022. URL : http://journals.openedition.org/medievales/637 ; DOI : https:// doi.org/10.4000/medievales.637

Ce document a été généré automatiquement le 23 avril 2022.

Tous droits réservés 


\title{
Le rôle des traductions médiévales dans l'évolution de la langue française et la constitution de sa grammaire
}

\author{
The Role of Mediaeval Translations in the Evolution of the French Language and \\ the Establishment of its Grammar
}

Claude Buridant

1 L'évolution de la langue française, depuis ses premiers linéaments, s'est accompagnée progressivement d'un ensemble de plus en plus étoffé d'instruments didactiques qui ont tenté d'en circonscrire le fonctionnement, d'en décrire les structures, d'en élaborer les règles. Mais à côté de ces instruments, les traductions ont souvent joué un rôle non négligeable dans l'élaboration d'une grammaire implicite, en servant de révélateurs de phénomènes majeurs touchant en particulier la syntaxe et témoignant de tendances évolutives profondes qui concernent son architectonique.

L'appareil grammatical : les ouvrages didactiques théoriques et pratiques

Dans l'évolution de la langue française, on s'est interrogé depuis longtemps sur le rôle joué par la formation de ses scripteurs au sens large et des instruments qu'ils avaient à leur disposition, soit les ouvrages didactiques grammaticaux, théoriques ou pratiques, les ouvrages lexicographiques sous toutes leurs formes, ou les traités proprement rhétoriques. Un ensemble d'études récentes ont mis en relief plusieurs phénomènes d'importance : la constitution de supports didactiques en langue vulgaire transférés du latin ; la découverte d'exercices pratiques de traduction à fonction didactique.

La constitution de supports didactiques en langue vulgaire transférés du latin On connaît le succès des deux artes rédigés par Donat au IV siècle, l'Ars minor et l'Ars maior, deux panoramas grammaticaux qui, intégrant les acquis des générations précédentes, fournissent un aperçu des parties du discours - dont le modèle remonte à la Techné grammatiké de Denys le Thrace -, augmenté, dans le cas de l'Ars minor, d'un 
aperçu des lettres, des syllabes, de barbarismes et de figures de style. Les panoramas grammaticaux de Donat sont devenus les modèles du traité grammatical pour les siècles postérieurs en servant de base aux premières descriptions des langues vernaculaires dans le monde celtique et dans le monde roman. P. Swiggers en donne les traits fondamentaux et dresse le bilan des premières grammaires vernaculaires françaises, dont les traités grammaticaux qualifiés d'« allocentriques ", dans la mesure où ils s'insèrent dans une production linguistique à orientation pratique ayant pour centre l'Angleterre. Parmi ces traités se place le Donat français rédigé en 1400, que P. Swiggers analyse pour souligner qu'il correspond à un moment de conversion et de transformation dans l'histoire de la tradition : tout en voulant enseigner les structures d'une langue vivante en voie d'expansion culturelle, et pour laquelle il s'agit d'établir une norme, il est surtout l'objet d'une attitude réflexive à propos du modèle grammatical, attitude marquée par un souci de systématisation dont témoigne la présence d'une section morphologique précédant l'analyse des parties du discours, articulée en deux volets théoriques. Un autre facteur de transformation (ou d'adaptation) est la reconnaissance de structures propres à la langue vernaculaire, en particulier par sousemploi (évacuation des verbes déponents), élargissement (construction impersonnelle l'on dit/on dit, pronoms possessifs, etc.) et abandon du modèle latin en faveur d'une description nouvelle, comme celle de la description du verbe, dont on distingue les deux manières, personnelles et impersonnelles. L'Ars minor de Donat devient ainsi « un dispositif descriptif, mais il est adapté aux structures de la langue vernaculaire et il est transformé pour répondre aux nouvelles exigences de systématicité $»^{1}$. M. ColomboTimelli, en réunissant dans une édition critique dix représentants des traductionsadaptations de l'Ars minor en ancien et moyen français, souligne aussi pour sa part leur importance pour l'approche grammaticale de la langue vulgaire: "L'analyse des huit parties du discours proposée par Donat, ainsi que la métalangue qui l'exprime, pèseront de tout leur poids sur la grammaire du français, auquel sont appliqués dès le début non seulement les termes techniques conçus pour le latin, pour la plupart simplement calqués et adaptés au système phonétique français, mais aussi le cadre théorique, ce qui sera lourd de conséquences pour l'analyse des aspects de la langue vulgaire absents ou différents du latin, de l'article notamment $»^{2}$. Et plus loin : « Même si le français ne fait jamais l'objet autonome d'une réflexion linguistique, on ne peut contester que nos traductions révèlent des traces importantes d'une conception qui voit dans le français, sinon une langue grammaticale à proprement parler, au moins une langue qui peut trouver une systématisation théorique, parfois au prix de quelques efforts d'adaptation, dans le cadre de la grammaire du latin. Sans que l'opposition entre latin/langue grammaticale et français/langue maternelle au sens le plus littéral du mot, soit jamais réellement mise en cause, la conception d'une possibilité de grammaire pour la langue vulgaire s'affirme peu à peu, et ceci, presque paradoxalement, dans des manuels destinés à l'enseignement de la langue ancienne $»^{3}$. Depuis l'étude fondamentale de T. Städtler, on peut mesurer à présent la fixation précoce et l'étendue insoupçonnée de la terminologie grammaticale en langue vulgaire, qu'on pensait à tort créée au $\mathrm{XVI}^{\mathrm{e}}$ siècle ${ }^{4}$.

La découverte d'exercices pratiques de traduction à fonction didactique

4 En concentrant ses études sur l'enseignement du français en Angleterre médiévale, A. Kristol a souligné pour sa part que «les grammairiens et professeurs des $\mathrm{XIV}^{\mathrm{e}}-\mathrm{XV}^{\mathrm{e}}$ siècles sont tout à fait à l'aise lorsqu'il s'agit de décrire par exemple la morphologie nominale ou verbale. Comme le montrent le Donat français ${ }^{5}$ et le Liber Donatib ${ }^{6}$ ils 
disposent d'une terminologie bien développée qui leur permet de distinguer par exemple les principaux tiroirs $d u$ système verbal; cette nomenclature provient évidemment des grammaires latines en langue vulgaire [...]. Par contre, dans l'ensemble des matériaux didactiques connus, il n'existe aucun manuel conçu pour l'enseignement de la syntaxe ». Même si certaines divergences entre la grammaire latine et celle du français sont correctement perçues et identifiées, les grammairiens anglais du Moyen Âge ne disposent encore d'aucun outil notionnel et terminologique qui leur aurait permis d'expliciter des problèmes comme l'ordre des mots ou l'emploi des temps et des modes en français. Même dans la mesure où une réflexion syntaxique au sujet du latin existe déjà au Moyen Âge, le transfert et l'application concrète de ces notions à la langue vivante ne semble pas avoir eu lieu. D'où les questions posées par A. Kristol : « De quelle manière [...] les intellectuels, les aristocrates et les bourgeois anglais du Moyen Âge apprenaient-ils non seulement à faire des phrases convenables en français élémentaire, à un niveau de langue enseigné par les manières de langage, mais à passer à un niveau de compétences supérieur? Quel est l'enseignement qui leur permettait d'apprécier des textes littéraires français [...] et même d'en rédiger eux-mêmes ? Et de quelle manière les étudiants d'Oxford parvenaient-ils aux compétences linguistiques avancées dont ils avaient besoin pour pouvoir accéder à l'enseignement théorique en grammaire et en orthographe qui leur était destiné ? $\gg^{7}$. Or, dans cette terra incognita, A. Kristol nous livre le précieux témoignage d'un document didactique contenu dans le ms. $188 \mathrm{du}$ Magdalen College d'Oxford dont la deuxième partie est consacrée entièrement à un grand exercice de traduction sur un fragment de la Somme le Roi, composé en 1280 par frère Laurent, confesseur du roi Philippe le $\mathrm{Hardi}^{8}$. Le texte original français est accompagné de deux traductions, en latin et en moyen anglais, présentant une vraie complémentarité, surtout dans le domaine du lexique, mais aussi dans le domaine de la morphologie et de la syntaxe. La fine analyse d'A. Kristol révèle qu'il s'agit là d'une "traduction "didactique" " : le texte latin a une fonction didactique fondamentale; en fonctionnant comme ajout, il permet de mettre en relief, au mépris même de sa syntaxe parfois, les phénomènes grammaticaux les plus caractéristiques de la langue source, en l'occurrence le français. En dehors du lexique, les deux traductions permettent ainsi d'expliciter de plusieurs manières la structure morphologique ou syntaxique du français, tel le sujet indéterminé «l'en" pour lequel les traducteurs cherchent à explorer toutes les équivalences possibles en anglais et en latin.

Mais l'un des enseignements majeurs est de mettre en relief la "grammaire floue » de l'ancienne langue que j'ai soulignée à plusieurs reprises9. Celle-ci est illustrée : d'abord par la nature bifrons de l'infinitif, en tension constante entre le plan verbal et le plan nominal, i.e. pouvant connaître des emplois allant jusqu'à la substantivation pleine du côté nominal et jusqu'au verbe personnel du côté verbal, les emplois mixtes n'étant pas rares en ancien français, où l'infinitif substantivé régit un complément ${ }^{10}$. A. Kristol relève ainsi, dans une énumération qui contient une série de sept infinitifs, six infinitifs perçus et rendus par des noms en latin et en anglais. Deuxième phénomène, la nature incertaine de l'adverbe, pouvant être réinterprété comme adjectif ou comme nom sous la plume du traducteur, comme dans l'exemple suivant, où le texte français fait une énumération des biens matériels et spirituels que Dieu fait à l'homme $\left(\mathrm{f}^{\circ} 36 \mathrm{v}^{\circ}-37 \mathrm{r}^{\circ}\right)$ :

De par l'ame. cler senz pur bien entendre pur bien trover bone memoire pur ben retenir.

On the soule byhalve clere witte for to understonde good for to fynde goode good mynde for to holde good thynge. 
Ex parte anime. clarum ingenium propter bonum intelligere bona memoria propter bonum invenire bona memoria propter bene retinere retinere

Si la traduction latine interprète à juste titre ben (forme anglo-normande de bien) comme un adverbe (« une bonne mémoire servant à bien mémoriser »), en anglais, en revanche, la structure change, et ben est rendu par un adjectif : for to holde good thynge " pour retenir de bonnes choses ». « On mesure ici, conclut A. Kristol, l'incertitude du traducteur anglais face aux catégories grammaticales floues de l'ancien français, et les vertus de la double traduction qui fonctionne comme un révélateur de cette incertitude ${ }^{11}$.

Plusieurs enseignements peuvent être tirés de ce qui précède : à côté des ouvrages didactiques visant à une description réglée de la langue française et acquérant une portée réflexive, il existe bien des exercices pratiques, des « exercitations » mettant en jeu le fonctionnement de la langue in vivo pour des locuteurs ayant le français comme langue seconde ; ces « exercitations " s'opèrent dans des traductions, en l'occurrence le latin et l'anglais dans le cas de la Somme le Roi, qui jouent le rôle de tests, le latin étant sans doute une pierre de touche privilégiée comme langue de référence ${ }^{12}$; au regard des ouvrages didactiques, qui comportent une large part de raidissement méthodologique, ces traductions mettent en relief une grammaire floue semblant assez différente.

Le rôle des traductions dans l'évolution de la langue française : une grammaire implicite

8 Le rôle de pierre de touche du latin par rapport à la langue vernaculaire, s'il est à l'œuvre dans ce style d'exercices, est bien évidemment fondamental dans les traductions médiévales, et il a été mis en relief à de nombreuses reprises après les timides observations de $\mathrm{F}$. Brunot ${ }^{13}$. À cet égard, les observations $\mathrm{d}^{\prime} \mathrm{Y}$. Lefèvre à propos de la traduction de l'Elucidarium sont toujours d'une lumineuse pertinence :

On admettra aisément que la traduction est un cas tout à fait exceptionnel de l'expression des idées : c'est le cas où la pensée s'est constituée indépendamment de la langue dans laquelle elle doit prendre forme, celui où la pensée, par sa structure et ses concepts, dépasse donc presque toujours les cadres habituels de cette langue, où l'écrivain doit pousser jusqu'à leurs limites extrêmes ses possibilités d'expression, puis adapter ce qui dans la pensée est irréductible à la langue dont il use ; c'est, de plus, le seul cas où il est possible de connaître, grâce à la version originale, la pensée que l'écrivain s'est proposé d'exprimer, autrement que par l'expression qu'il a employée. On comprend, par conséquent, que les traductions offrent un champ de recherche privilégié à qui veut déterminer à leur juste valeur les moyens d'expression qu'une langue met à la disposition de ceux qui l'utilisent ${ }^{14}$.

9 Dans ma thèse, j'ai souligné pour ma part, dans le même sens, à quel point les traductions du latin en français peuvent jouer un rôle de révélateur, au sens photographique du terme, des tendances profondes de la langue-cible, c'est-à-dire, en l'occurrence, du français ${ }^{15}$. C'est ce même rôle que met en relief C. Brucker en 1977 en traitant de «La valeur linguistique des traductions médiévales» à propos des « constructions infinitives en moyen français " ${ }^{16}$.

10 Cette fonction révélatrice est susceptible de mettre en évidence les résistances, les dominances et les émergences morphosyntaxiques et lexicales de la langue-cible, selon les distinctions opérées par G. Antoine sur le plan stylistique - distinctions reprises, avec des affinements, de R.-L.Wagner ${ }^{17}$. Par dominance, G. Antoine entend les faits syntaxiques régnants par opposition à la résistance et à l'émergence : la première est la 
survivance de faits syntaxiques anciens résistant à la pression de faits nouveaux; la seconde est l'apparition de faits syntaxiques nouveaux. Soit le schéma :

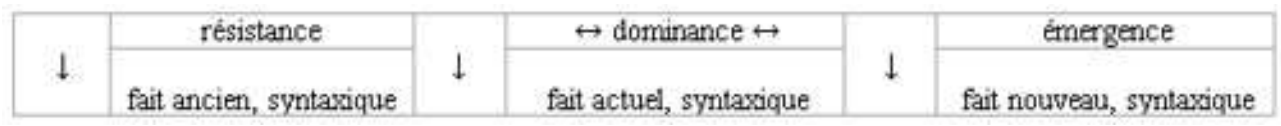

11 Ce schéma, pour peu qu'on y inclue la morphologie et le lexique, peut s'appliquer au phénomène de la traduction qui nous occupe ici, la question étant de savoir dans quelle mesure la langue source, en l'occurrence le latin, se heurte à la résistance de la languecible, étaye sa dominance, ou favorise l'émergence de faits linguistiques nouveaux.

En ce sens, C. Brucker montre bien que la construction infinitive de type savant, dans la traduction du Policraticus de Jean de Salisbury en 1372 par Denis Foulechat (qui appartient à l'équipe des traducteurs de Charles V), participe de l'émergence de tout un ensemble de formes nominales en moyen français.

13 Comme il l'observe en effet, la construction infinitive savante ${ }^{18}$ est sans doute « devenue, pour le traducteur, un moyen d'expression indispensable qu'il voudrait voir utiliser systématiquement dans la langue, au point qu'il en fait un emploi qui, jugé de l'extérieur, peut paraître excessif $»^{19}$, mais replacé dans l'histoire de la langue, cet emploi stylistiquement marqué est compatible avec les tendances profondes de la syntaxe du moyen français: l'exploitation de la construction infinitive de type savant par Denis Foulechat s'inscrit dans la promotion des formes nominales du verbe qui entretiennent avec cette construction des relations extrêmement étroites dans leur emploi même. La traduction, ici comme souvent, "a un effet de grossissement, à deux titres au moins : d'une part, le traducteur qui se heurte à des difficultés d'équivalence peut se voir obligé de recourir à un outil grammatical jusqu'alors laissé en marge de la langue courante et même à forcer l'emploi de cet outil ; d'autre part, une tendance latinisante peut être considérée comme une sorte d'exagération révélatrice d'un état latent $\aleph^{20}$. Cette émergence ténue peut se repérer déjà dans la traduction des Décrets de Gratien, sous la pression du latin, dans des exemples relevés par L. Löfstedt ${ }^{21}$. Le mouvement de latinisation constitue un réseau de formes nominales contribuant à étager et à hiérarchiser la phrase en prose du moyen français au sens large, avec l'expansion des constructions absolues en participe présent ou passé prédicat, comme le relève J. Müller-Lancé à la suite de maintes études ${ }^{22}$.

Les résistances de la langue-cible

14 Le témoignage des traductions est aussi précieux pour ce qu'il peut nous révéler des points de résistance de la langue-cible et les renouvellements qu'elle est amenée à consentir sous la pression de ce "fort latin» dont parlent les traducteurs, en soulignant par là trois points majeurs: sa richesse, sa puissance de concision synthétique et sa puissance d'abstraction. Ce sont ces éléments que je voudrais aborder à présent, en prenant quelques points d'observation remarquables illustrés d'exemples empruntés à la vaste panoplie des traductions médiévales, compte tenu de leur genre et de leur visée, deux éléments déterminants en l'occurrence.

Le « dépliage paratactique »

15 L'importance du genre a sans doute été soulignée depuis longtemps : le latin n'offre pas les mêmes difficultés au traducteur selon qu'il s'agit de chroniques ou de textes narratifs écrits dans un style simple et sans aspérités ou de textes théoriques et réflexifs maniant le raisonnement et l'abstraction. Y. Lefèvre note que la langue du traducteur anonyme transposant en français au $\mathrm{xIII}^{\mathrm{e}}$ siècle l'Elucidarium, œuvre 
d'Honorius Augustodunensis composée sans doute vers le début du XII ${ }^{\mathrm{e}}$ siècle et visant à mettre à la portée des laïcs une théologie pratique, est « dès le premier abord, beaucoup moins bien adaptée à l'expression des idées abstraites ${ }^{23}$. Un exemple particulièrement net de cette distinction et de l'impact qu'elle peut avoir sur la traduction est bien celui de Jean de Vignay, qui s'est exercé dans ces deux types de textes, sans avoir nécessairement les capacités d'un excellent latiniste. D. Gerner, éditeur de sa traduction des Otia imperialia, peut ainsi observer que «les passages construits sur une base essentiellement narrative trouvent volontiers à se couler dans des phrases, dans des paragraphes, rédigés dans une syntaxe qui suggère le naturel et le spontané d'une langue authentique». En revanche, c'est dans les passages didactiques à dominance abstraite, philosophique, théologique, exégétique, ou dans les parties scientifiques ou techniques "que surgissent le plus souvent ces phrases embarrassées, à la syntaxe défectueuse et empêtrée de latinismes, réunies en paragraphes dans lesquels la continuité thématique n'est que très rarement assurée, quand elle ne disparaît pas tout à fait " $^{24}$. Et j'écrivais dans le même sens : "Alors que pour les développements philosophiques, Jean de Vignay calque péniblement la lourde syntaxe étagée du latin, il retrouve dans cette partie (i.e. la troisième partie des Oisivetés), des patrons de phrase familiers de séquences narratives. La construction de la phrase française est relativement aplanie par rapport à la construction étagée du latin, la multiplication de l'adverbe si thématisant soulignant les articulations de ces séquences [...]. Dans la transposition tendent aussi à s'imposer des articulations narratives en quant.../ si, qui répondent à un souci stylistique, mais sont aussi révélateurs d'une architectonique mentale [...] tendant à ne laisser aucun vide dans le récit, de le suivre "en continu" dans une chaîne d'actions et de réactions en thèmerhème, comme l'a souligné $B$. Combettes ${ }^{25}$. Un autre phénomène intéressant, bien que ténu, repérable chez Jean de Vignay, est l'apparition, dans les séquences narratives, de propositions temporelles de perception jouant le rôle de liant, répondant éventuellement aux adverbes du latin comme itaque, proinde ${ }^{26}$. On observe de manière générale que les structures hypotaxiques du latin qui subsistent dans les chroniques originales sont volontiers dénouées et mises à plat dans les traductions au profit d'un enchaînement continu de phrases coordonnées: c'est un des traits marquants de la traduction de La Chronique des rois de France, à travers ses deux témoins, dont celui du manuscrit Vatican Reg. lat. $624^{27}$. Il y a là un procédé de dépliage, sur un plan étale, de l'hypotaxe de l'original. Ce dépliage n'est pas exceptionnel et semble même un phénomène marquant la plupart des traductions médiévales du latin, au moins jusqu'au XIV ${ }^{e}$ siècle. L. Löfstedt l'observe aussi pour sa part dans son étude syntaxique sur un passage de la traduction française des Décrets de Gratien, où elle rassemble un faisceau de traits concordant ${ }^{28}: 1^{\circ}$ la résolution des subordonnées latines en principales; $2^{\circ}$ l'efflorescence, en revanche, de subordonnées françaises représentant des participes latins, dont le plus souvent des relatives (adjectives), mais aussi des temporelles. Cette tendance à l'étalement paratactique du latin n'est sans doute pas particulière au français ${ }^{29}$.

16 Sur cette base, conclut L. Löfstedt, il apparaît que l'ancien français se serait déjà « emparé, au sein de la famille romane, de la place accordée aujourd'hui au français, à l'italien et au catalan par K. H. Körner : il préfère les constructions au verbe fini $»^{30}$, ce que confirme P. Stein dans son étude sur les traductions du De urbe condita de Tite-Live, en dégageant en conclusion les tendances diachroniques du français quant à la concurrence entre formes finies et formes non finies des constructions verbales dans 
leurs différentes fonctions syntaxiques ${ }^{31}$. C'est surtout à mesure que les traductions se développeront dans le domaine de la philosophie et de la réflexion abstraite que s'accentuera la tendance à manier l'hypotaxe, à organiser les phrases en étagement en recourant aux formes nominales d'arrière-plan comme les propositions participes, les transferts du latin, comme l'ablatif absolu.

L'équivalence polysémique

Les phénomènes d'émergence et de résistance s'observent aussi dans le domaine du lexique. Les traductions favorisent en moyen français l'émergence d'un vocabulaire abstrait, comme l'a parfaitement montré $\mathrm{S}$. Heinimann ${ }^{32}$, l'un des procédés de transposition des traducteurs étant de faire passer en français un mot de facture savante venant du latin en l'appuyant par la glose d'un mot courant qui lui sert en quelque sorte d'appui et de "passeport » dans l'itération synonymique, comme je l'ai souligné à plusieurs reprises ${ }^{33}$. Mais les traducteurs peuvent aussi avoir affaire à un lexique particulièrement riche et varié, transcrit par des mots de large polysémie, et cette polysémie est en quelque sorte un facteur de résistance du fait de la large palette d'acceptions qui lui permettent de rendre sans néologismes les mots latins correspondants, en utilisant ici encore les ressources du binôme synonymique. L'excellent lexique de C.Brucker, annexé à son édition du Policratique, permet d'apprécier ce propos que peut illustrer l'exemple significatif de faux ${ }^{34}$. Il répond à :

incredulus (III, 9, 32) : « qui n'a pas la foi »

pravus, dans le couple « fausse et mauveise volenté » (II, 27, 87) : " pervers »

dubius, dans le couple «fausse et doubteuses» (Prol. P. 51): "qui relève de

l'erreur"

falsus (II, 4, 28 ; Prol. P 36 ; II, 4, 24 ; 14, 3) : « erroné »

Il est épaulé en quelque sorte par fausseté, souvent appuyé d'un doublet qui oriente son sens :

« fausseté » = falsitas (II, 18,$4 ; 27,39 ;$ III, 5) : « perfidie »

« fausseté - desloiauté » : perfidie (II, 27, 188)

« fausseté - trahison »= iniquitas (III, 4, 7)

«fausseté - vilté » = perfidia (III, 6, 12)

Les particules séparées

Des éléments de résistance et d'émergence peuvent aussi se repérer, à travers les traductions, dans le domaine de la morphologie, comme peuvent l'illustrer les exemples suivants où le latin joue le rôle de révélateur. On sait en effet que l'ancien français emploie volontiers un vaste jeu de "particules séparées » analogues aux particules séparables de l'allemand, insérées dans la catégorie floue des adverbesprépositions, qui, comme dans les doublets synonymiques, renforcent le sens du verbe qu'elles accompagnent ou souvent orientent le sens de verbes au large spectre sémantique, les «verbes incolores» («farblose Verben»), comme les appelle S. Heinimann ${ }^{35}$. Les traductions emploient fréquemment ce jeu de particules, caractéristique de fortes tendances analytiques, pour rendre l'éventail des préfixes latins qui n'émergeront que très progressivement, ainsi qu'en témoignent les ouvrages lexicographiques de la Renaissance. Le phénomène peut être illustré par la traduction des Décrets de Gratien, dans des exemples relevés par leur éditrice L. Löfstedt dans le glossaire de son édition ${ }^{36}$ :

Particule arrieres, qui peut traduire l'idée de repousser, de retour ou de répétition :

Bouter arrieres: depelli debuerunt (D 22 c 5, 1. 3) / removendi sint > «doivent estre boutés arrieres » (C 3 q 4, 1. 2) - Ego repellam te, ne sacerdotio fungaris michi > « Ge te bouterai arrieres que tu n'uses d'ordre de provoire » (D 38 c 6, 1. 12). 
Giter arrieres: Abjecto proposito > « qui ont gité arrierres (le proposement) » (C 27 q1 $\mathrm{c} 11,1.3)$ / postponere > « mettre arriere dos » (C $11 \mathrm{q} 3 \mathrm{c} \mathrm{8}, 1.12)$.

Demander arrieres : Lex Iulia repetundarum $>$ « la loi que Iulius fist demander arrieres ce qui est donné pour mauvese cause » (C 1 q 1 c 17, l. 1).

Baptizier arrieres : rebaptizari non debent > « ne doivent pas estre baptizié arrieres » (DC D 4 c 129, 1. 25).

Fort ensemble de particules avec le verbe largement polysémique mettre:

Mettre avant : crimen illatum > « le crime qu'il a mis avant » (C 2 q 3 c 5, 1.8).

Mettre devant : nullum crimen eis obici potest > « ne leur puet nus crimes estre mis devant » (C 3 q 1 c, 1. 4, Glossaire, p. 458) ; opponebantur > « estoit mise devant » (C 23 q 6 c 3, 1. 13).

Mettre hors : extre castra positus > « (il estoit) mis hors d'avec les autres » (C 11 q 3 14, 1.5).

Mettre sus : alii inferre > "mettre sus autre " (D 45 c 4, 1. 11); tibi ab adversario tuo obiciuntur « tes aversaires te met sus » (C 3 q $11 \mathrm{c} \mathrm{4,} \mathrm{l.} 1$ ); crimen imposuerit (C 6 q 2c 1, 1. 2).

La dérivation préfixale

Un autre point de résistance de la morphologie dérivationnelle est la dérivation préfixale en in- du latin, d'abord rétive à la transposition synthétique, non seulement en français, mais dans d'autres langues romanes comme l'espagnol, qui lui préfèrent la "négation syntactique », selon les termes d'E. Llorens ${ }^{37}$. J'ai largement développé ce point en traitant des Problèmes d'équivalence pour les adjectifs et les adverbes en in- ${ }^{38}$, où sont regroupées des observations faites par d'autres chercheurs, tel I. Short, éditeur de la Chronique du Pseudo-Turpin de William de Briane, signalant la traduction de: incognitum (qua beati tempori corpus latabat incognitum, I, 1. 51) > « mes nen ne le savoit mie " (I, 1. 72); innumeras (ceterasque regiones innumerasque urbes, I, 1. 42) > « et autres régiouns et cités dount il n'est nul numbres » $(\mathrm{I}, 1.61)$; innumerabilis (Clamor quoque et luctus et vox plangentium innumerabilis erat, XXVI, 1. 84) > « unkes teles ne fu vou ne oyes (Ly cry et la noyse comensa si graunt ke unkes teles ne fu vou ne oyes) », XXVI, l. 259-260), etc. ${ }^{39}$ J'y notai pour ma part que les Lexiques français sont par ailleurs un sûr témoin de ce manque: les différentes versions d'un des Lexiques latin-français du Moyen Âge édités par M. Roques, l'Abavus ${ }^{40}$, rendent ainsi ineffabilis ("ineffable») par "c'on ne puet dire", impugnabilis par "c'on ne puet vaintre» (ms. de Douai, 62), indicibilis par "que l'en ne puet dire » (ms. du Vatican, lat. 2748), comme le relève R.L. Wagner en observant que « là où les humanistes, du XIV ${ }^{\mathrm{e}} \mathrm{au} \mathrm{XvI} \mathrm{I}^{\mathrm{e}}$ siècle, n'hésiteront pas à former des latinismes, les glossateurs de l'Abavus préfèrent commenter et expliquer au moyen d'une relative ou autrement des mots dont la structure se prêtait à une francisation $»^{41}$. C'est progressivement que commencent à se créer les correspondants en in- des adjectifs négatifs latins en in- + base + ilis en particulier ${ }^{42}$. L'Aalma en offre un exemple à côté des formes en non: intolerabilis ( $\left.{ }^{\circ} 6154\right)$ est rendu par "intolerables" à côté de "non soufrables", mais ineffabilis est rendu par "non racontables ", inenarrabilis par « ce qui ne peut estre raconté », etc. Dans la plupart des œuvres de ce siècle, les périphrases sont encore en grande majorité et l'Ovide moralisé, traduction libre des Métamorphoses d'Ovide, offre ainsi, pour instabilis : «Ensi n'estoit pas la terre estable », et pour innabilis: « ne la mer n'estoit pas noable» (I, v. $16:$ Sic erat instabilis tellus, innabilis unda).

Dans ma thèse, j'ai fait le bilan des palliatifs utilisés en les comparant partiellement aux propositions de Lexiques d'autres œuvres ${ }^{43}$. Soit: $1^{\circ}$ Les équivalences des nombreux dérivés en in-/-im + base + (b)ilis ou (b)iliter : inaccessibilis, incomprehensibilis, ineluctabilis, inexplicabilis, inexpugnabilis, inflexibilis, infragabiliter, inhabitabilis, immobilis, immortalis, 
innumerabilis, impartabilis, imperviabilis, implacabilis, immortabilis, impoossibilis, instabilis, insufficienter, insuperabilis, intolerabilis, inutilis, invadalis, irrecuperabilis-irrecuperabiliter, irrevocabilis-irrevocabiliter. $2^{\circ}$ Les autres adjectifs en in-: illibatus et inconvulsus, immeritus, immunis, impatiens, improvidus, impunis, inauditus, incertus, incognitus, incompertus, indebitus, indemnis, indignus, inefficax, infamatus, infectus, infidus, infinitus, inhumanus, inhumatus, injuste, immemor, inmisericordis, innocens, inobs, inopinatus, inscius, insensatus, insolitus, insufficienter, intactus, invia, invitus.

Pour les adjectifs de la première catégorie, c'est sans doute le noyau « c'an ne puet »+ procès actif / " qui ne puet » + procès passif qui s'impose le plus souvent, avec toutes les transformations possibles, à côté de «neant» + adjectif en -able («neant departable ", «neant profitable»); mais d'autres types se dégagent: "sans » + base verbale («sans estre pris », "sans plus recovrer », «sans renuier »); « sans » + base nominale («sans fin », « sans faille», « sans habiteors »). Des solutions plus ponctuelles sont également notables (correspondant sémantique large comme « fiers » pour rendre mente immobilis par exemple : $c f$. infra).

Ce relevé permet donc d'apprécier un large jeu des équivalences utilisées dans cette traduction. Les lexiques n'offrent, somme toute, qu'un clavier restreint de correspondances, de définitions théoriques. Dans la réalité du discours, des périphrases variées rendent les composés latins, se ramenant pour la plupart à un certain nombre de types fondamentaux. Un exemple comme inexpugnabilis est particulièrement illustratif ${ }^{44}$.

Qua muris inexpugnabilibus munitissima erat (Pseudo-Turpin de Compostelle) > « quar ele estoit molt bien garnie de murs qui nul essaut ne doutoient » (Pseudo-Turpin du ms. Vat. Regina 624) / «car bien estoit garnie de paiens et de tres fors murs» (Pseudo-Turpin du ms. BnF fr. 2137) / « car ele ert garnie de tres forz murs » (PseudoTurpin de Johannes).

Castrum munitissimum vallo arto et rigido, superius glande, inferius torrentis profunditate pene inexpugnabilis (Vie de Louis VI) > «et par ce estoit tiex (le chastel) que a bien pres qu'il n'avoit garde ne d'escu ne de lance » (Vie de Louis VI, version A du ms. Vat. Regina 624).

Ut parte ex omni sit castrum inexpugnabile (Guillaume le Breton, Philippide) > « et que de toutes pars fust deffansables et garnis » (i.e. le château) (Philippide).

Turribus et muris nimis altis atque profundis. / Fossis interior burcus securior ab omni. / Hoste videbatur, et inexpugnabilis esse (Guillaume le Breton, Philippide) > « et de grans murs et de hautes tors et de parfons fossés sambloit estre fors li bors defors, et sans estre pris» (Philippide); Abavus : inexpugnabilis : «c'on ne puet vaintre ".

Une large palette d'équivalents se retrouve dans d'autres textes témoins, comme la traduction des Décrets de Gratien, enregistrés par le Commentaire et le Glossaire de L. Löfstedt ${ }^{45}$ :

Correspondant de large spectre sémantique : impius > «fel/felon »; rex inpiissimus > «li feus rois » (c 27, 1. 1).

Équivalent en non-, qui semble le procédé le plus courant dans les substantifs : inpossibilitas offitii > « la nom puissance de fere l'office charnel » (C $33 \mathrm{q} \mathrm{I,} \mathrm{1.} \mathrm{17).}$

Équivalent par une proposition relative dans les adjectifs ou les adverbes, qui "déplie» à des degrés divers, en quelque sorte, la préfixation synthétique de l'original : inculpabile : dicimus dimittendum et inculpabile iudicandum > «nous disons [...] que l'en doit lessier et ne doit l'em pas blasmer » $(\mathrm{Q} 1 \mathrm{c1}, 1.9)^{46}$. du latin ont eu tendance à n'employer, pour les adjectifs en in-, qu'un jeu restreint 
d'équivalences mécaniques, dont essentiellement «neant» + les correspondants de la base latine ${ }^{47}$. Entre ces calques et l'apparition, au XIV siècle, des dérivés savants en inmarquant un progrès dans la morphologie dérivationnelle ${ }^{48}$, il $\mathrm{y}$ a place pour une gamme de possibilités exploitées par les traducteurs pour s'adapter aux situations contextuelles, dont la Chronique des rois de France est un témoin intéressant.

La dérivation suffixale

Dans le domaine de la dérivation, les traducteurs se heurtent aussi à la panoplie des noms d'agent latins en -ator. La question a également été examinée dans ma thèse, où à partir d'un relevé systématique, j'ai dégagé les tendances suivantes ${ }^{49} .1^{\circ}$ Répondent au latin un ensemble de noms d'agent en -eor renvoyant à une fonction bien déterminée dans les domaines les plus divers : en général largement répandus à l'époque (au XIII ${ }^{\mathrm{e}}$ siècle), ils sont indépendants du mot latin qu'ils sont chargés de rendre et l'identité de base qu'ils ont éventuellement avec leur correspondant est fortuite (cf. "ardeor", " artifieor ", « combateor ", « correor », « minaor ", « piqueor», " poigneor », «crieor ", "gaigneor» (des terres). $2^{\circ}$ Répondent en second lieu au latin tout un ensemble de cas où les noms d'agent en -eor de l'ancien français sont employés dans le cadre d'une définition lorsqu'est requise une caractérisation abstraite. Dans ces cas, la traduction tend à répondre aux sollicitations du latin par des correspondants en -eor bien attestés mais parfois aussi par de véritables calques qui font figure d'hapax, comme dans cet exemple: liberalis ditator et elemosinarum dapsilis dispensator ${ }^{50}>$ " anrichisserres d'eglise et liberaus ammoniers " ${ }^{51}$. Deffandeor est largement utilisé en équivalence du latin (dans des contextes du même type) comme épithète fondamentale de nature : princes, prélats et rois reçoivent les titres de « deffandeors de la foi, de la Sainte Église, du royaume $»^{52}$. Il est remarquable qu'en face de cet exemple, defensor ou synonyme - au sens de "défenseur militaire, d'une place assiégée en particulier ", soit le plus souvent rendu par « cil qui / çaus qui » + procès verbal circonstancié, dont voici deux exemples, parmi d'autres : defensores > « cil qui le chastel deffandoient " (Vie de Louis VI) / "le chastelain» (Grandes chroniques); defensores turris > "çaus qui la [la tour] gardoient », et plus loin, turrim defendentes > "çaus qui la desfendoient » (Vie de Louis VI) ${ }^{53}$.

Il y a donc dans ce cas, peut-on conclure, une large concurrence de la construction périphrastique : il semble que, en face de la suffixation en -eor emportant avec elle un minimum d'abstraction, de concentration formelle, cette construction favorise mieux l'ancrage dans le concret grâce à la relative et à son procès : « çaus qui la tor/le mur/le chastel deffandent» plutôt que "*li deffandeor de la tor». La transposition périphrastique est en tout cas un procédé commode en lieu et place d'un correspondant synthétique du latin. Remarquable est, à cet égard, le rôle de la proposition relative, qui permet de transcrire "par dépliage » le contenu d'un mot, comme dans cet exemple tiré de la Chronique des rois de France : cunctis in circuitu nationibus > « lé gens de toutes les contrees qui environ ceulx marchissoient » / " toutes les autres nacions $"$ "

La traduction du neutre latin

Un autre point sensible amenant les traducteurs à chercher des ressources originales dans la langue-cible est la traduction du neutre latin. Le problème est évidemment récurrent. Y. Lefèvre le relève dans ses remarques sur la traduction du Lucidaire au XIII ${ }^{\mathrm{e}}$ siècle pour l'emploi neutre de l'adjectif; le traducteur a alors recours au nom " chose » : bonum = «bone chose " (I 191, II 5); sua = « les seues choses" (I 199); grave = "grief chose» (II, 4). Et il ajoute: «Si l'on songe à l'usage que le traducteur fait de 
"chose" dans les périphrases qui lui servent à rendre diverses conjonctions, on comprend que ce substantif, peu significatif par lui-même, inonde le texte français $~^{55}$. C'est aussi le même mot qui rend le neutre latin dans la traduction des Décrets de Gratien, comme le relève L. Löfstedt en distinguant son emploi comme élément grammatical et son emploi en sens plein, à propos d'un exemple tiré du De Consecratione: "Utilisés dans la même phrase, "choses" n'est qu'un élément grammatical pour traduire un neutre latin "les autres choses" (cetera), alors que "chose" est un mot plein dans l'expression "en chose" correspondant au latin in re ${ }^{56}$. Le mot y traduit différents types de neutre :

Substantif : asperum... et inimicum > " aspre chose et contraire" (C 25 Q 2 C 7) ; quid autem iniquius est quam impia sapere > " quel chose est plus felonnesse que savoir felonesses choses » (C 24 Q 3 C 30) bon exemple de la traduction du neutre latin.

À côté d'équivalents: «li jugemenz» (masc.)... « ou les choses» (fém.)... « ou celles» (fém.), traduction de decreta (neutre)... siue que... seu que...la traduction de decreta étant jugée peu heureuse par L. Löfstedt (C 25 Q 2).

Interrogatif, pour la traduction de quid: quid autem iniquius est quam impia sapere > « quel chose [...]» (cf. exemple supra); mais aussi sui sint sortilegi > « quel chose est sorcerie » (C 26).

Cet aperçu ne saurait évidemment rendre compte de tout ce que révèlent les traductions médiévales sur l'évolution de la langue française et la constitution de sa grammaire. Dans ce modeste essai, on n'a voulu évoquer que quelques aspects non négligeables de cet apport. Il semble bien que, par rapport au latin, les traductions médiévales se situent à un carrefour de tension entre dépliage et concentration.

Dépliage, sur le plan typologique, dans la traduction, de la forte condensation synthétique du latin et de son fort coefficient d'abstraction par un ensemble d'équivalents analytiques qui se situent aux différents étages de la langue, qu'il s'agisse de la syntaxe, de la morphologie et même du lexique : décomposition analytique de la syntaxe, rendant volontiers par des enchaînements étales la forte hypotaxe du latin, processus d'explicitation par des relatives, binômes synonymiques, morphèmes externés répondant aux éléments compositionnels du latin. Autant de phénomènes qui sont des éléments de résistance de la langue-cible tout en l'obligeant à exploiter au maximum ses ressources.

Concentration cependant dans l'évolution des traductions vers l'exploitation de ressources plus synthétiques, dont l'on a évoqué au passage quelques témoignages : structures hypotaxiques en syntaxe, formation des mots par affixation, dans une latinisation qui n'est pas nécessairement un copie directe du latin mais qui peut être une "latinisation interne", comme dans le cas des propositions infinitives de type savant définies ci-dessus, moyens affinés de rendre l'abstraction. Autant d'éléments qui se développent à la faveur de la mise en français de textes savants en latin, dont l'un des promoteurs majeurs est Nicole Oresme : à la faveur de ses traductions, précisément, il «fait éclater l'opposition solidement ancrée dans les esprits de son temps entre le latin, langue savante et langue de grammaire, et le français, langue maternelle apprise par imitation. D'ailleurs, la traduction ne constitue dans sa perspective que le premier moment dans le processus d'acquisition par le français du registre de l'expression savante $»^{57}$. À travers ce registre le français tend à renouveler sa grammaire et son lexique dans le sens de la hiérarchisation et de l'abstraction, fortement corrélés ${ }^{58}$.

Si toute traduction engage l'historicité de la langue, si son enjeu est un élargissement de la capacité d'une langue, donc d'une culture (et d'une nation), à faire sens et à 
gagner en plasticité, à accroître son pouvoir de dire, comme le souligne Humboldt dont les réflexions sur le traduire - "das Übersetzen »- sont une source inépuisable de méditations ${ }^{59}$, les traductions médiévales dans leur ensemble offrent un observatoire privilégié pour apprécier précisément l'évolution de la langue médiévale et la constitution d'une grammaire au sens d'un ensemble d'instruments et de struments conditionnant et réglant son fonctionnement.

\section{NOTES}

1. P. Swiggers, «La tradition de l'Ars de Donat et les premières grammaires vernaculaires du français : un moment de conversion », dans Zur Geschichte der Grammatiken romanischer Sprachen, W. Dahmen, G. Holtus, J. Kramer, M. Metzeltin et P. Wunderli éd., Tübingen, 1991, p. 153.

2. M. Colombo-Timelli, Traductions françaises de l'Ars minor de Donat au Moyen Âge (XIIIXVe siècles), Florence, 1996, p. 8-9.

3. Ibid., p. 11.

4. T. STÄDTLER, Zu den Anfängen der französischen Grammatiksprache, Tübingen, 1988.

5. Ibid., p. 128-37.

6. The Liber Donati. A Fifteenth-Century Manual of French, B. MERRILEES et B. SITARZFITZPATRICK éd., Londres, 1993, p. 8 sq.

7. A. Kristol, «Le ms. 188 de Magdalena College Oxford : une "pierre de Rosette" de l'enseignement médiéval du français en Angleterre? », Vox Romanica, 60, 2001, p. 150-51 (je souligne).

8. Le texte français est malheureusement encore inédit. E. Brayer a consacré sa thèse de l'École Nationale des Chartes (1940) à l'édition d'une des versions de La Somme le Roi. Est attendue dans la collection de la Société des Anciens Textes Français l'édition préparée de longue date par elle-même et $\mathrm{F}$. Leurkin, mais dont le corpus ne comprendrait pas le présent manuscrit, lacunaire et corrompu. Ce dernier a fait l'objet d'un mémoire de maîtrise par $\mathrm{C}$. Nissille, Prolégomènes à une analyse linguistique. Manuscrit Oxford Magdalen 188, un support d'enseignement du français en Angleterre élaboré par un traducteur face à ses propres limites régionales et diachroniques, Neuchâtel, Faculté des Lettres, 2000. C. Nissille projette d'approfondir ces Prolégomènes dans une thèse. 9. Cf. C. Buridant, «Varietas delectat. Prolégomènes à une grammaire de l'ancien français ", Vox Romanica, 55, 1996, p. 88-125, et Id., Grammaire nouvelle de l'ancien français, Paris, 2000.

10. Ainsi, dans cet exemple, la préposition introduisant l'infinitif peut en même temps servir de support à l'article du substantif régi dans une construction double : « As dras vestir plus ne repose,/Si a les sa mere laissiez » (Il n'hésite pas un seul instant à revêtir ces habits, abandonnant ceux donnés par sa mère) (Conte du Graal, ms. T, v. 1622-23). C'est un point que j'ai abordé dans « L'infinitif dans les langues romanes et les langues germaniques : essai d'approche typologique ", dans Toward a Typology of European Languages, Berlin-New York, 1990, J. Bechert, G. Bernini, C. Buridant ed., Empirical Approaches to Language Typology, 8, p. 141-63. J'y reviendrai largement dans un essai en 
préparation aux éditions Champion, La substantivation de l'infinitif en français : aperçu diachronique.

11. A. Kristol, « Le ms. 188 de Magdalen College Oxford », loc. cit., p. 165.

12. Sur ce point, $c f$. les réflexions orchestrées dans l'introduction de ma Grammaire nouvelle de l'ancien français, op. cit., p. 29-30, et le tableau récapitulatif de la p. 40.

13. Rappelées par $C$. Brucker au début de son article, « La valeur du témoignage linguistique des traductions médiévales : les constructions infinitives en moyen français ", dans Linguistique et philologie, Amiens, 1977, p. 325-44.

14. Y. Lefèvre, «La traduction du latin par un clerc français au XII siècle », dans VIII Congresso di Studi romanzi, Florence, 1956, p. 219.

15. C. Buridant, Les Problèmes de traduction du latin en français au XIII siècle à partir de l'Histoire de France en français de Charlemagne à Philippe-Auguste, Thèse dactylographiée, Université de Lille III, 1978.

16. C. Brucker, «La valeur du témoignage linguistique des traductions médiévales », loc. cit., p. 325.

17. G. Antoine, La Coordination en français, Paris, 1959, t. I, p. 64.

18. Sous ce type savant, il faut ranger $: 1$. La construction infinitive dépendant de verbes de déclaration ou de volonté, très rare dans l'ensemble en ancien français ; 2 . la construction infinitive dont le verbe à l'infinitif est estre ou un verbe affecté d'une forme composée d'infinitif (passive en général), même si le verbe qui la régit est un verbe de perception comme veoir ; 3 . la construction infinitive avec syntagme « substantif + infinitif » sujet ou objet (suivi d'un attribut) du verbe principal ou en tournure personnelle ou impersonnelle. (C. Brucker, « La valeur du témoignage linguistique des traductions médiévales », loc cit., p. 342, note 6 et p. 329-31.)

19. Ibid., p. 332.

20. Ibid., p. 339.

21. La Concordia discordantium canonum, somme théologique compilant les canons de l'Église et base du droit canonique jusqu'en 1918, est traduite en français avant 1170 par un clerc anonyme, sans doute à l'instigation de Thomas Beckett, pour les seigneurs laïcs de l'empire des Plantagenêts. L'édition latine en a été fournie par Friedberg, Decretum magistri Gratiani, Leipzig, 1879. La traduction en ancien français est publiée par L. Löfsfedt, Gratiani Decretum. La traduction en ancien français du Décret de Gratien. Édition critique, Helsinki (5 vol. : I, Distinctiones, 1992 ; II-IV, Causae, 1993-1997 ; V, Observations et explications, 2001). L. Löfstedt relève ainsi un exemple de proposition infinitive dépendant d'un verbe de déclaration calquée sur le latin : « (dixit) quatuor (esse) genera divinationum, terram, aquam [...] » (C 26, Q 3 et 4, Commentaire linguistique 1. 3, vol. V, p. 346).

22. J. MÜLLER-LANCÉ, Absolute Konstruktionen vom Altlatein bis zum Neufranzösischen, Tübingen, 1994. L'auteur prend cependant essentiellement en compte, sous « moyen français », le français de la Renaissance, n'évoquant qu'incidemment, par un seul exemple, le français du $\mathrm{Xv}^{\mathrm{e}}$ siècle.

23. Y. Levèvre, Une traduction du XIII siècle: Le Lucidaire, thèse complémentaire, Université de Paris, Faculté des Lettres, 1954, p. 99. Ces remarques ont été considérablement développées et affinées dans les deux éditions récentes de quelques versions de la traduction de l'Elucidarium : la version $1:$ «Lucidaire de grant sapientie ». Untersuchung und Edition der altfranzösischen Übersetzung 1 des « Elucidarium " von Honorius Augustodunensis, M. KLEINHANs éd., Tübingen, 1992 ; les versions 2, 4 et 5 : «Lucidaire 
vault tant a dire comme donnant lumiere ». Untersuchung und Edition der Prosaversionen 2, 4 und 5 des « Elucidarium », M. KLEINHANs éd., Tübingen, 1993.

24. D. Gerner, La traduction des Otia imperialia de Gervais de Tilbury par Jean de Vignay dans le manuscrit Rotschild $n^{\circ} 3085$ de la Bibliothèque nationale de Paris. Édition et Étude, thèse dactylographiée, Strasbourg, 1995.

25. B. Combettes, Recherche sur l'ordre des éléments de la phrase en moyen français, thèse de doctorat d'État, Nancy, Atelier National de Reproduction des thèses, Université de Lille III, Lille, 1988. Rappelons qu'en termes de communication d'un message, le thème peut être défini comme ce à propos de quoi une information est communiquée, et le rhème comme le nouvel élément communiqué.

26. C. Buridant, «La traduction du latin au français dans les encyclopédies médiévales à partir de l'exemple de la traduction des Otia imperialia de Gervais de Tilbury par Jean de Vignay et Jean d'Antioche ", Translation Theory and Practice in the Middle Ages, J.

M. Beer éd., Kalamazoo, 1997, p. 156-59.

27. Relevé dans ma thèse, Les Problèmes de traduction du latin en français au XIII ${ }^{e}$ siècle, op. cit., t. I.

28. L. Löfstedt, «Étude syntaxique de la traduction française du Decret de Gratien », Neuphilologische Mitteilungen, 99, 1998, p. 313-41 (notamment p. 323 et 323-25).

29. P. Andersen l'observe dans une communication récente au colloque Pratiques de traduction au Moyen Âge (Institut d'Études Romanes de l'Université de Copenhague, 25-26 octobre 2002) sur la traduction d'une version latine du Roman d'Alexandre par Wichbolt, vers la fin du Moyen Âge, où l'on retrouve de manière frappante les caractéristiques du récit paratactique, sous l'effet possible de la « surtraduction ». La traduction de Wichbolt est étudiée par P. Andersen dans sa thèse soutenue à l'Université Jules Verne d'Amiens en 2000 : L'Alexandre du Groner Seelentrost, l'Alexandre de Wernigerode et l'Alexandre de Wichbolt, trois adaptations allemandes du Pseudo-Callisthène. Je remercie P. Andersen pour les renseignements qu'il m'a communiqués à cette occasion.

30. L. Löfstedt, "Étude syntaxique de la traduction française du Décret de Gratien », op. cit., p. 323. La référence est à K. H. Körner, Korrelative Sprachtypologie. Die zwei Typen romanischer Syntax, Stuttgart, 1987, p. 60-84. Dans ce recueil d'articles, K. H. Körner distingue deux grands types de langues romanes sur la base d'un certain nombre de phénomènes syntaxiques fortement corrélés : au premier type appartiennent le français, l'italien central et le catalan, au second le portugais, l'espagnol et le roumain. Parmi les traits spécifiques du premier type, il faut compter la tendance à l'emploi du verbe fini, i.e. conjugué personnel, là où le second type a recours plus volontiers aux formes non-finies, i.e. nominales, comme l'infinitif. L'ensemble des traits est bien résumé par C. Hernandez Sacristan dans le compte rendu de l'ouvrage paru dans Vox Romanica, 49-50, 1990-91, p. 438-40.

31. P. STEIN, Untersuchungen zur Verbalsyntax der Liviusübersetzungen. Ein Versuch zur Anwendung quantitativer Methoden in der historisch-vergleichenden Syntax, Tübingen, 1997, p. 247.

32. S. Heinimann, Das Abstraktum in der französischen Literatursprache des Mittelalters, Bern, 1963.

33. $C f$., entre autres, $C$. Buridant, « Les binômes synonymiques. Esquisse d'une histoire des couples de synonymes du Moyen Âge au xvii ${ }^{\mathrm{e}}$ siècle ", Bulletin du Centre d'Analyse du discours, 4, 1980, p. 5-79. 
34. Denis Foulechat, Le Policratique de Jean de Salisbury (1372). Livres I-III, C. BRUCKER éd., Genève, 1994, p. 403-04.

35. S. Heinimann, Das Abstraktum in der französischen Literatursprache des Mittelalters, op. cit., p. 87 sq. Cf. aussi C. Buridant, « Les particules séparées en ancien français », dans Romanistique-Germanistique : une confrontation, C. Buridant éd., Strasbourg, 1987, p. 185-204; et C. Buridant, Grammaire nouvelle de l'ancien français, op. cit., chap. 14. 36. L. LÖFSTEDT, Gratiani Decretum, op. cit., vol. V, Glossaire, p. 433, 429 et 458.

37. E. Llorens, La Negacion en espanol antiguo, con referencias a otros idiomas, Madrid, 1929, p. 201.

38. C. Buridant, Les Problèmes de traduction du latin en français au XIII siècle, op. cit., t. II, Livre III, chap. IV.

39. I. SHORT, The Anglo-Norman Pseudo-Turpin Chronicle of William de Briane, Londres, 1966, p. 163. Thèse à la base de son édition, The Anglo-Norman Pseudo-Turpin chronicle of William de Briane, Oxford, 1973. C'est à cette édition que renvoient les références en regard du texte latin établi par A. Hämel et A. De Mandach, Der Pseudo-Turpin von Compostela, Munich, 1965.

40. M. Roques, Recueil général des lexiques français du Moyen Âge (XII ${ }^{e}-X V^{e}$ siècles), I. Lexiques alphabétiques, Paris, 1936-1938 (2 vol.). Cette œuvre présente deux lexiques alphabétiques latin-français désignés par le premier mot (Abavus et Aalma) qui ouvrent chacun d'eux. La collection a été augmentée depuis par l'édition d'un ensemble de lexiques parus dans la série Lexica Medii Aevi. Nouveau recueil de lexiques latin-français du Moyen Âge (Turnhout), soit : le Dictionarius de Firmin Le Ver (Abbeville, 1544), B.

Merrilees et W. Edwards éd., 1994 ; le Glossarium gallico-latinum (1430-1450), B. Merrilees et J. Monfrin éd., 1998 ; le Vocabularius familiaris et compendiosus de Guillaume le Talleur, Rouen, (c. 1490), W. Edwards et B. Merrilees éd., 2002. Une version de l'Aalma est désormais disponible sur la toile, avec un moteur de recherche (http:// www.chass.utoronto.ca/ merrilee/aalma.htm).

41. R.-L. Wagner, Les Vocabulaires français. I. Définitions. Les dictionnaires, Paris, 1967, p. 101

42. Cf. E. Thorne-Hammar, Le Développement de sens du suffixe latin-bilis en français, LundCopenhage, 1942. Sur la concurrence, dans les lexiques, entre la dérivation en -able, " populaire » ou francisante, et la dérivation en -ible, « savante » ou latinisante, selon la distinction qu'elle établit, $c f$. la communication de B. Merrilees à la Journée d'Étude organisée à Strasbourg par le Centre de Linguistique et Philologie romane de l'Université Marc Bloch et l'Équipe SILEX de l'Université Charles de Gaulle - Lille III, sur La Morphologie dérivationnelle dans l'ancienne langue française et occitane : « La morphologie dérivationnelle en français médiéval : l'apport des lexiques ", Actes à paraître dans le $\mathrm{n}^{\circ} 17$ de Lexiques.

43. C. Buridant, Les Problèmes de traduction du latin en français au XIII siècle, op. cit., II, III, chap. IV.

44. Cf. Der Pseudo-Turpin von Compostela, II, 1. 86-87, A. Hämel éd., Münich, 1965, p. 42 ; Pseudo-Turpin du ms. Vatican Regina 624, II, 1, C. Buridant éd., Genève, 1974, p. 88 ; La chronique dite de Turpin. Publiée d'après les mss. BN 1850 et 2137, 1. 27-28, F. Wulff éd., Lund, 1881 , p. 44 ; The Old French Johannes translation of the Pseudo-Turpin chronicle : a critical edition, VIII, 3-4, R. N. Walpole éd., Berkeley-Los Angeles, 1976 ; Vie de Louis VI le Gros, chap. XI, H. Waquet éd., Paris, 1964, p. 72 ; Vie de Louis VI le Gros. Version A du ms. Vatican Regina 624, X, 13, C. Buridant pré-éd. et Grandes chroniques, J. Viard éd., Paris, 1920-53, t. V, p. 131 ; Guillaume le Breton, Philippide, I, v. 608 et II, v. 553-555, dans CEuvres de Rigord 
et Guillaume le Breton, historiens de Philippe-Auguste, H.-F. Delaborde éd., Paris, 1882-85 (2 vol.) ; Philippide, I, 95C et II, 81, C. Buridant pré-éd. ; Abavus I, III, IV-V, dans M. Roques, Recueil général des lexiques français, op. cit.

45. L. Löfstedt, La Traduction française des Décrets de Gratien, vol. V, op. cit., p. 448, 460 et 362.

46. P. Nobel relève pour sa part deux équivalents de impudicus dans le Décret, à l'appui de l'étude succincte, mais dense, qu'il consacre à la traduction : « Il se présente un certain nombre de cas où le correspondant savant n'est pas encore attesté à l'époque de la traduction. Les translateurs du XIV siècle, se plaignant de la pauvreté du vocabulaire français, auront tout naturellement recours, dans ces cas-là, à l'emprunt latin, créant ainsi de nombreux néologismes qui enrichiront le vocabulaire français. Telle n'est pas la pratique dans notre texte : Ab ecclesiae eliminandae et ergastula sunt monachorum vel monacharum impudicae personae (C. 27, 1. c. 11) > " moines et nonains qui ne gardent cloistre doivent estre mis en chartre" ; Inpudicas detestabilis personas (ibid.) > "desleaus persones". C'est l'inexistence d'impudique au XIII siècle qui nous vaut cette transposition » (P. Nobel, « La traduction médiévale du Décret de Gratien », Revue de droit canonique. Le Décret de Gratien revisité. Hommage à Rudolf Weigand, 48/2, 1998, p. 335). 47. C'est bien ce qu'offre, par exemple, le Psautier de Cambridge dans sa glose interlinéaire continue : l'index-glossaire de l'édition F. Michel enregistre 16 occurrences de nient + adjectif/adverbe, comme incessabilis > « nient cessable », increduli > « nient creable », etc.

48. Cf. E. Staaff, « Étude sur les mots composés avec le préfixe négatif in- en français ", Studia Neophilologica, 1928, p. 45-73.

49. C. Buridant, Les Problèmes de traduction du latin en français au XIII siècle, op. cit., t. II, p. 531-541.

50. Vie de Louis VI le Gros, op. cit., p. 192.

51. Vie de Louis VI le Gros, version A du manuscrit Vatican regina 624, XXVI, op. cit., p. 33. Ces noms d'agent à haute valeur caractérisante se concentrent dans le planctus sur la mort de Roland dans le Pseudo-Turpin du manuscrit Vatican Regina 624, op. cit., p. 115.

52. Grandes chroniques de France, op. cit., t. V, p. 35.

53. Vie de Louis VI le Gros, version A du manuscrit Vatican regina 624, op. cit., X, 14, VIII, 16 et 17 ; et Grandes chroniques de France, op. cit., t. V, p. 35.

54. De l'origine troyenne jusqu'à l'année 829 , I, C. Buridant prééd., I, V, 20, et Grandes chroniques de France, I, op. cit., t. I, p. 17.

55. Y. Lefèvre, Une traduction du XIII siècle : Le lucidaire, op. cit., p. 111.

56. L. LÖFSTEDT, Gratiani Decretum, op. cit., vol. V, p. 422. Les références sont données au Commentaire, chapitre IV dans ce volume, respectivement p. 343, 339, 343 et 345.

57. S. Lusignan, « La topique de la Translatio studii et les traductions françaises de textes savants au XIV ${ }^{\mathrm{e}}$ siècle ", dans Traduction et traducteur au Moyen Âge, G. Contamine éd., Paris, 1989, p. 312. Cette acquisition se manifeste aussi dans le lexique.

58. Sur le plan lexical, « la contribution de Nicole Oresme à l'évolution du lexique de la langue française moderne est énorme », comme le note M. Heinz en conclusion de ces "Quelques remarques sur la néologie dans les traductions de Nicole Oresme ", qui relève que 300 à 350 néologismes sont sans aucun doute attestés pour la première fois chez lui : Cahiers de publications doctorales, Université Marc Bloch, numéro spécial Horizons de la linguistique romane. Actes de la Journée de Linguistique Romane (25 avril 
2002), recueillis par F. Pierno, p. 65. La syntaxe des ouvrages scientifiques, quant à elle, mériterait encore des études approfondies.

59. W. von Humboldt, Sur le caractère national des langues et autres écrits sur le langage, présenté, traduit et annoté par D. Thouard, Paris, 2000.

\section{RÉSUMÉS}

L'évolution de la langue française, depuis ses premiers linéaments, s'est accompagnée progressivement d'un ensemble de plus en plus étoffé d'instruments didactiques qui ont tenté d'en circonscrire le fonctionnement, d'en décrire les structures, d'en élaborer les règles. Mais à côté de ces instruments, les traductions ont souvent joué un rôle non négligeable dans l'élaboration d'une grammaire implicite, en servant de révélateurs de phénomènes majeurs touchant en particulier la syntaxe, mais aussi le lexique, et témoignant de tendances évolutives profondes qui touchent son architectonique, la traduction étant au carrefour de résistances et d'émergences de la langue-cible (le français) entre dépliage explicatif et concentration synthétique.

The Role of Mediaeval Translations in the Evolution of the French Language and the Establishment of its Grammar. The evolution of the French language, right from its very first outlines, was progressively accompanied by a more and more ample ensemble of didactic instruments which attempted to circumscribe its function, to describe its structures and to elaborate its rules. Alongside these instruments, however, translations often played a non-negligible role in the elaboration of its implicit grammar, in revealing major phenomena that touched in particular on its syntax but also on its vocabulary. Translation, lying as it does at the crossroads of the resistance to and the emergence of the targeted language (French), between an explicative unfolding and a synthetic concentration, showed in this way evidence of the profound evolutionary tendencies that touched on its architectonics.

\section{INDEX}

Keywords : latin, Old French, grammar, linguistics, translation

Mots-clés : traduction, ancien français, grammaire, linguistique

\section{AUTEUR}

\section{CLAUDE BURIDANT}

Université de Strasbourg-II-Marc Bloch, 22, rue René Descartes, Strasbourg-Esplanade, F-67084

Strasbourg Cedex 